\title{
Diagnosis of orthopaedic-implant-associated infections caused by slow-growing Gram-positive anaerobic bacteria - a clinical perspective
}

\author{
Diana Salomi Ponraj ${ }^{1}$, Thomas Falstie-Jensen ${ }^{2}$, Nis Pedersen Jørgensen ${ }^{3}$, Christen Ravn ${ }^{4}$, \\ Holger Brüggemann ${ }^{5}$, and Jeppe Lange ${ }^{1,6}$ \\ ${ }^{1}$ Department of Clinical Medicine, Aarhus University, Aarhus, 8000, Denmark \\ ${ }^{2}$ Department of Orthopaedic Surgery, Aarhus University Hospital, Aarhus, 8200, Denmark \\ ${ }^{3}$ Department of Infectious Diseases, Aarhus University Hospital, Aarhus, 8200, Denmark \\ ${ }^{4}$ Department of Orthopaedic Surgery, Lillebaelt Hospital, Kolding, 6000, Denmark \\ ${ }^{5}$ Department of Biomedicine, Aarhus University, Aarhus, 8000, Denmark \\ ${ }^{6}$ Department of Orthopaedic Surgery, Horsens Regional Hospital, Horsens, 8700, Denmark
}

Correspondence: Jeppe Lange (jeppe.lange@ @lin.au.dk)

Received: 28 May 2021 - Revised: 7 September 2021 - Accepted: 13 September 2021 - Published: 7 October 2021

\begin{abstract}
Slow-growing Gram-positive anaerobic bacteria (SGAB) such as Cutibacterium acnes are increasingly recognized as causative agents of implant-associated infections (IAIs) in orthopaedic surgeries. SGAB IAIs are difficult to diagnose because of their non-specific clinical and laboratory findings as well as the fastidious growth conditions required by these bacteria. A high degree of clinical suspicion and awareness of the various available diagnostic methods is therefore important. This review gives an overview of the current knowledge regarding SGAB IAI, providing details about clinical features and available diagnostic methodologies. In recent years, new methods for the diagnosis of IAI were developed, but there is limited knowledge about their usefulness in SGAB IAI. Further studies are required to determine the ideal diagnostic methodology to identify these infections so that they are not overlooked and mistakenly classified as aseptic failure.
\end{abstract}

\section{Introduction}

Implant-associated infections (IAIs) in orthopaedic surgery, which includes prosthetic joint infections (PJIs) in this review, are associated with high morbidity and mortality (Tande and Patel, 2014; Fischbacher and Borens, 2019) and remain a highly strenuous scenario to encounter for most orthopaedic surgeons and patients alike. Aerobic bacteria, primarily staphylococcal species, are the most identified organisms in culture-positive IAI. Slow-growing Gram-positive anaerobic bacteria (SGAB) are increasingly being recognized as potential agents in IAI, although their role is not well established, and their diagnosis is challenging (Tande and $\mathrm{Pa}$ tel, 2014; Shah et al., 2015; Bjerke-Kroll et al., 2014; Singh et al., 2012; Achermann et al., 2014; Aubin et al., 2014; Portillo et al., 2013; Lin et al., 2020). The purpose of this narrative review is to introduce and highlight all SGAB, including the rarer ones; to evaluate current diagnostic SGAB-IAI information from a clinical perspective, helping to guide the diagnostic process; and to identify areas for future research.

\section{SGAB-IAI case report}

A 68-year-old man presented with complaints of persistent pain from a primary total hip arthroplasty (THA), which had been performed due to idiopathic osteoarthrosis 3 years prior. The immediate post-operative period had been uneventful, but during the following 2 years he experienced increasing hip pain. Due to a medical evaluation of general malaise and chronic elevated serum erythrocyte sedimentation rate (ESR), an ${ }^{18}$ F-FDG PET/CT $\left({ }^{18}\right.$ F-fluorodeoxyglucose positron emission tomography/computed tomography) was performed, which showed increased tracer uptake around the THA, and he was referred to the corresponding author's hip 
clinic. He had elevated ESR and mildly raised serum Creactive protein (CRP). Ultrasound-guided hip joint aspiration was performed, and analysis showed a total white blood cell (WBC) count of 55540 cells per $\mu \mathrm{L}$ and polymorphonuclear neutrophils (PMNs) of 52170 cells per $\mu \mathrm{L}$. However, no growth was found in culture after $14 \mathrm{~d}$ of incubation on two consecutive ultrasound-guided hip joint aspirations.

In view of the patient's symptoms, imaging, and laboratory findings, prosthetic joint infection (PJI) was suspected based on modified Musculoskeletal Infection Society (MSIS) criteria (Falstie-Jensen et al., 2019a), and revision of the THA was planned as per the CORIHA protocol (Lange et al., 2018). By the time the patient was admitted for revision surgery, he had developed a sinus tract from the THA to the skin, which in all classifications is a definite sign of infection. During the one-stage revision, five tissue biopsies were obtained as recommended by Kamme and Lindberg (1981). No growth was seen in any of the cultures after $14 \mathrm{~d}$ of standardized incubation.

Sonication of the implant was performed as part of an unrelated research project. Finally, Cutibacterium acnes (C. ac$n e s)$ was isolated from the sonicated fluid after $6 \mathrm{~d}$ of anaerobic incubation, with no growth presented in the aerobic cultures. The patient was treated as per local hospital guidelines for management of PJI. The patient had no pain or other sign of PJI recurrence at latest follow-up and had an eventless, contralateral, THA in the follow-up period due to idiopathic osteoarthrosis. The patient has given full written consent for the use of his case.

\section{Slow-growing Gram-positive anaerobic bacteria: definition and species}

SGAB comprise Gram-positive bacteria of the two phyla Firmicutes and Actinobacteria that are usually slow-growing and require long cultivation times ( $>3 \mathrm{~d}$ but often longer) when isolated from clinical samples. Their growth relies on anaerobic metabolism with limited or no growth under aerobic conditions. SGAB species that have been reported as causative agents of IAI are listed below.

Cutibacterium species (previously Propionibacterium): these Gram-positive, aerotolerant, anaerobic coryneform bacteria are part of the normal skin microbiota. The most common species associated with IAI are $C$. acnes (Lutz et al., 2012; Bossard et al., 2016) followed by C. avidum (Renz et al., 2018a).

C. acnes: although reported as a causative agent of IAI since the 1970s, it has gained more interest in recent years as a biofilm-producing pathogen (Kamme and Lindberg, 1981; Achermann et al., 2014). It is the most common anaerobic bacterium isolated in IAI and is most commonly seen in late onset, chronic PJI (Lebowitz et al., 2017; TriffaultFillit et al., 2019). It has a predilection for the upper limb and the spine and is considered to be the most common or- ganism causing shoulder PJI (Namdari et al., 2019; Sevelda and Fink, 2018; Achermann et al., 2013; Zhang et al., 2015; Marmor et al., 2016; Kerneis et al., 2017), but it is also found in PJI of hip, knee, and metacarpophalangeal joints, as well as spinal IAI (Drago et al., 2017; Nodzo et al., 2016; Bacle et al., 2017; Garg et al., 2015; Sampedro et al., 2009). It is also the most common bacterium grown in unexpected positive cultures in revision shoulder arthroplasty and removed clavicle plates (Padegimas et al., 2017; Foruria et al., 2013; Lucas et al., 2016; Both et al., 2018; Gausden et al., 2017). C. acnes is a normal skin commensal. In patients undergoing primary shoulder surgery, it was found that Cutibacterium species were cultured from unprepared epidermal skin surfaces and freshly incised dermal edges in $72 \%$ and $34 \%$, respectively (Matsen et al., 2020). Studies have also reported the presence of Cutibacterium species in deep tissue cultures in patients undergoing primary shoulder arthroplasty despite thorough skin preparation and preoperative intravenous antibiotic prophylaxis (Matsen et al., 2015; Hudek et al., 2014).

C. avidum: it is the second most common Cutibacterium species associated with IAI (Zeller et al., 2007, 2018; Wildeman et al., 2016; Renz et al., 2018a). It is mostly seen in PJI of the hip, especially when the anterior approach is used, but infections involving fixation plates of the femur, humerus, and prosthetic shoulder joints have also been reported (Renz et al., 2018a; Zeller et al., 2018; Sampedro et al., 2009; Achermann et al., 2017; Boni et al., 2018).

C. granulosum: the first case of PJI due to this bacterium was described in 2013 in a patient after THA (Nystrom et al., 2013; Zeller et al., 2018).

Finegoldia magna (previously Peptostreptococcus mag$n u s)$ : this Gram-positive anaerobic coccus is part of the commensal flora, found on healthy human skin and as a part of the gut microbiota (Söderquist et al., 2017; Levy et al., 2009). Although less frequent than Cutibacterium species, PJIs of hip, shoulder, knee, and foot, as well as osteosynthesisrelated IAI due to F. magna, have been reported (Levy et al., 2009; Söderquist et al., 2017; Renz et al., 2018a; Achermann et al., 2013; Walter et al., 2014; Richards et al., 2014; Kamme et al., 1974; Puchner et al., 2017; Akgun et al., 2020; Rieber et al., 2020; Anagnostakos et al., 2021).

Staphylococcus saccharolyticus (previously Peptococcus saccharolyticus): this anaerobic coagulase-negative staphylococcus has been isolated from a limited number of cases of shoulder and hip PJI and spinal IAI (Trojani et al., 2020; Evans et al., 1978; Pumberger et al., 2019; Brüggemann et al., 2019; Söderquist et al., 2021).

Parvimonas micra (previously Micromonas micros or Peptostreptococcus micros): a few cases of IAI due to this Grampositive anaerobic coccus have been reported to date (Renz et al., 2018a; Rieber et al., 2018, 2020; Bartz et al., 2005; Stoll et al., 1996; Z. Huang et al., 2019; Anagnostakos et al., 2021).

Miscellaneous SGAB: in addition to the above, IAI due to rare SGAB like Slackia exigua, Robinsoniella peoriensis and 
Facklamia hominis have also been reported in case reports (Rieber et al., 2016, 2019; Corona et al., 2014). With recent focus on Cutibacterium acnes and its role in IAI, many studies have been done on that species. But there is limited information on rarer SGAB, even though their role in IAI is not of any less importance.

\section{The diagnosis of SGAB IAI}

\subsection{Classification}

Regarding the definition of PJI, different classifications have been proposed by the Musculoskeletal Infection Society (MSIS), the Infectious Diseases Society of America (IDSA), and the European Bone and Joint Infection Society (EBJIS) (Parvizi et al., 2011, 2018; Osmon et al., 2013; Renz et al., 2018b; McNally et al., 2021). Although there are differences, several criteria are present throughout all the guidelines, such as the presence of a sinus tract, histopathologic evidence of inflammation, and positive cultures from tissue biopsies or aspirations. In addition, evaluation of inflammatory cells and biomarkers, both in serum and synovial fluid, are also taken into consideration (Renz et al., 2018b; Parvizi et al., 2011, 2018; Osmon et al., 2013). Whether these criteria are stringently applicable for SGAB PJI remains uncertain, amongst others due to these organisms' specific growth requirements and in vivo reduced inflammatory response. Moreover, there are no established or widely accepted diagnostic criteria for non-PJI IAIs, making their diagnosis difficult and limiting the available data on non-PJI IAI, irrespective of the causative organism. As such, no clear classification has been established in regard to SGAB IAI.

\subsection{Clinical features}

Differentiation between SGAB IAI and aseptic failure based only on clinical symptoms is often extremely difficult, and a high degree of clinical suspicion is warranted, with pain being the most predominant feature.

Persistent or increasing pain from an area with an orthopaedic implant and joint stiffness in specific relation to PJI are the most common clinical symptoms reported in SGAB IAI. However, such symptoms are also frequently present in cases of aseptic failure (Renz et al., 2018a; Rienmuller and Borens, 2016; Jacobs et al., 2016; Wang et al., 2013; Hsu et al., 2018). The classical clinical features of IAI include fever, local signs of inflammation such as redness or warmth, pain and swelling, and a sinus tract. Fever and local signs of inflammation are not just non-specific but also observed less frequently in SGAB IAI than pain and joint stiffness (Rieber et al., 2016; Rieber et al., 2019; Corona et al., 2014; Maroto Piñeiro et al., 2021; Randall et al., 2020; Z. Huang et al., 2019). The frequency of a sinus tract in SGAB IAI has been reported anywhere from less than $1 \%$ up to $20 \%$ (Renz et al., 2018a; Rienmuller and Borens, 2016; Dodson et al., 2010; Jacobs et al., 2016).

Several groups have analysed the clinical significance of unexpected positive cultures (UPCs) of $C$. acnes in revision shoulder surgery and found that they had no clinical relevance in at least $25 \%$ of UPC cases (Foruria et al., 2013; Falstie-Jensen et al., 2021).

\subsection{Medical imaging}

Medical imaging is applied as routine first-line investigation in patients with orthopaedic implants. But plain radiographs are neither sensitive nor specific for the diagnosis of IAI, irrespective of the causative organism as it is difficult to differentiate between infection and aseptic loosening based on a single X-ray (Gemmel et al., 2012).

Only a few studies have distinctly described X-ray findings in SGAB IAI, with signs of loosening being the most frequent finding seen in $23 \%-73 \%$ of $C$. acnes IAI as well as in IAI due to less frequent SGAB (Renz et al., 2018a; Rienmuller and Borens, 2016; Wang et al., 2013; Hou et al., 2015; Pottinger et al., 2012; Rieber et al., 2016, 2019; Corona et al., 2014). However, it is difficult, if not impossible, to differentiate between aseptic and septic loosening (Lima et al., 2013) in these cases. Signs of osteitis, with osteolysis and/or periosteal bone formation, or abscess formation are some of the reported X-rays and ultrasonographic findings, respectively, of $C$. avidum-associated IAI (Zeller et al., 2018; Wildeman et al., 2016). Nuclear medicine imaging has not been found useful in the diagnosis of SGAB in chronic shoulder PJI (FalstieJensen et al., 2019b, c).

\subsection{Serum and synovial biomarkers}

In general, SGAB do not elicit a strong inflammatory response. Consequently, conventional inflammatory markers may not be elevated (Sampedro et al., 2009), and as such the value of existing serum and synovial biomarkers in SGAB IAI is questionable.

Several studies indicate that the WBC count is in the normal range in SGAB IAI (Renz et al., 2018a; Zappe et al., 2008; Sampedro et al., 2009; Nodzo et al., 2016) and that this also occurs in IAI due to more virulent organisms (Nodzo et al., 2016; Akgun et al., 2019). While ESR and CRP perform better compared to WBC count in the diagnosis of $C$. $a c$ nes IAI (Dodson et al., 2010), ESR/CRP cannot clearly differentiate between aseptic failure and SGAB IAI (Sampedro et al., 2009; Plaass et al., 2016). They are not always elevated in C. acnes IAI (Renz et al., 2018a; Figa et al., 2017), and when elevated, their levels are still lower compared to IAI caused by highly virulent microorganisms (Akgun et al., 2018; Grosso et al., 2014a; Nodzo et al., 2016). Normal ESR and CRP have also been reported in IAI caused by infrequent SGAB (Z. Huang et al., 2019; Corona et al., 2014). It should be noted that comparison between differ- 
ent studies is difficult, as different cut-off values were used for CRP (> $5 \mathrm{mg} \mathrm{L}^{-1}$; Plaass et al., 2016; Zeller et al., 2018; $>7 \mathrm{mg} \mathrm{L}^{-1}$; Dodson et al., 2010, or $>10 \mathrm{mg} \mathrm{L}^{-1}$ ) (Renz et al., 2018a; Nodzo et al., 2016; Grosso et al., 2014a; Akgun et al., 2018; Figa et al., 2017), while the latest EBJIS definition of PJI mentions that a CRP $>10 \mathrm{mg} \mathrm{L}^{-1}$ is suggestive of "likely infection" (McNally et al., 2021).

Synovial fluid and serum levels of interleukin-6 (IL-6) have been identified with a high sensitivity and specificity in the diagnosis of PJI of hip and knee in general, but little is known about their usefulness in SGAB IAI (Deirmengian et al., 2010; Alijanipour et al., 2013). Serum IL-6 has potentially a poor sensitivity and specificity and cannot currently be recommended as a preoperative diagnostic test in SGAB IAI (Frangiamore et al., 2015; Grosso et al., 2014b).

Synovial alpha defensins ( $\alpha$-defensin), an antimicrobial peptide, can be measured by enzyme-linked immunosorbent assay (ELISA) or by $\alpha$-defensin lateral flow (ADLF) tests and has been included in the recent EBJIS definition of PJI (Weigelt et al., 2020; McNally et al., 2021). In the diagnosis of $C$. acnes shoulder PJI, studies have shown that synovial $\alpha$ defensins by ELISA (Unter Ecker et al., 2019; Frangiamore et al., 2015) may have a role to play in $C$. acnes IAI, but Weigelt et al. (2020) found that the ADLF test failed to identify the only culture-positive $C$. acnes shoulder PJI in their study (Weigelt et al., 2020). Overall, it is difficult to assess the ADLF test's potential in SGAB IAI of hip and knee, as few or no SGAB were included in any of the studies that showed high sensitivity and specificity of the ADLF in hip and knee PJI (Deirmengian et al., 2014; Bonanzinga et al., 2017; Gehrke et al., 2018). Focus of future studies should be on evaluating the ELISA test in SGAB IAI.

The simple and inexpensive leukocyte esterase strip test (also used for urinary tract infections) has an overall concordance of $86 \%-93 \%$ with culture results in hip and knee PJI in general (Di Benedetto et al., 2019). But its use in SGAB IAI might be limited, as the test could only correctly identify 3 of 15 C. acnes shoulder PJIs (Unter Ecker et al., 2019).

Other biomarkers like serum D-dimer and synovial CRP are also included as minor criteria in an updated PJI definition (Parvizi et al., 2018). However, studies on D-dimer have shown conflicting results in IAI in general (Shahi et al., 2017; J. Huang et al., 2019), similarly contradictive results have been seen in the role of synovial CRP (Omar et al., 2015; Tetreault et al., 2014), and their use in SGAB IAI has not been evaluated. Serum procalcitonin has also previously been evaluated and not found to be of value in the diagnosis of PJI in general (Xie et al., 2017; Saleh et al., 2018).

\subsection{Synovial fluid cytology}

Aspiration of synovial fluid is often performed in suspected cases of PJI. When no lavage has been performed and clear fluid is aspirated, synovial fluid cytology gives valuable information about the level of local inflammation (McNally et al., 2021). Elevated synovial WBC count is regarded as a diagnostic criterion in all major definitions of PJI (McNally et al., 2021; Parvizi et al., 2018; Osmon et al., 2013). But different studies have mentioned different cut-offs. The latest EBJIS definition suggests synovial fluid WBC count of $>3000$ cells $/ \mu \mathrm{L}$ and the presence of $>80 \%$ PMNs as likely cut-offs for PJI of hip and knee (McNally et al., 2021). These values are not applicable in PJI of upper limbs and can also be affected by use of immunosuppressive drugs or presence of co-morbid conditions like inflammatory arthritis, metalon-metal prosthesis, or crystal-induced arthritis (Ottink et al., 2019).

However, synovial fluid WBC count is not always increased in SGAB IAI, and when elevated, their numbers are similar to that seen in more virulent microorganisms (Renz et al., 2018a; Nodzo et al., 2016). In C. avidum PJI, the median synovial WBC count was reported to be high with a median differential count of $95 \%$ PMNs (Zeller et al., 2018).

\subsection{Histopathology}

Histopathological examination revealing the presence of neutrophils is an important criterion in the overall diagnosis of PJI. In the 2021 EBJIS definition of PJI, the presence of $\geq 5$ neutrophils in $\geq 5$ high-power field (HPF or $400 \times$ magnification) or presence of $\geq 5$ neutrophils in a single HPF indicate confirmed or likely infection, respectively (McNally et al., 2021). However, there is a difference in histopathologic findings in IAI caused by $C$. acnes and $C$. avidum, with $C$. avidum causing a more robust immune response. Renz et al. (2018a) reported that inflammation was seen in peri-implant tissue in $100 \%$ of IAI caused by $C$. avidum, but when cases of $C$. acnes were included in the evaluation, the overall positivity dropped to $67 \%$ (Renz et al., 2018a). Histopathological examinations of intraoperative peri-implant tissue samples are frequently negative for the presence of neutrophils, with a positivity of only about $17 \%-50 \%$ in C. acnes-associated PJI (Figa et al., 2017; Sampedro et al., 2009; Butler-Wu et al., 2011; Grosso et al., 2014a).

Interestingly, Hudek et al. (2021) used immunohistochemistry with $C$. acnes-specific antibodies on tissue samples taken from patients undergoing primary shoulder surgery or arthroscopy for the first time and detected $C$. acnes intracellularly within stromal cells and macrophages (Hudek et al., 2021). While the results of their study point towards $C$. $a c$ nes being a commensal in the shoulder, immunohistochemistry can also be potentially used to diagnose SGAB IAI with histopathology.

\subsection{Microbiology}

Microbiology remains the most important clinical entity in IAI as this, besides confirming the diagnosis (McNally et al., 2021), also provides a potential antibiogram to help guide 
the clinician in the adjuvant antibiotic treatment following surgery. The methods can be culture dependent or culture independent. However, especially concerning SGAB IAI there, are many aspects to take into consideration for the clinician, as SGAB by nature demands sophisticated processing not always undertaken in an everyday clinical setting, unless the clinician is acutely aware of the potential presence of SGAB as the causative organism.

\subsubsection{Culture-dependent methods}

Culture-dependent methods are relatively cheap and readily available in any clinical microbiology laboratory. The success of culture-dependent methods depends on several factors, e.g. the nature of the organism to be isolated, the quality, type and number of specimens collected, and conditions and duration of incubation (Larsen et al., 2012). Samples for microbiological diagnosis of IAI can be obtained preoperatively or intraoperative, as aspirations or biopsies, but also following implant subjected to sonication (Zimmerli et al., 2004). In case of culture-negative PJI, culture-independent methods, such as next-generation sequencing (NGS), might be a useful addition to identify the causative SGAB (Tarabichi et al., 2018).

Aspiration appears most useful in C. avidum IAI with one study showing the growth of this bacterium in preoperative aspiration fluid in 14 out of 15 cases (Zeller et al., 2018). It has been found that $C$. acnes IAI culture of synovial/periimplant fluid was not diagnostically accurate and showed low sensitivity compared to sonication fluid and peri-implant tissue (Renz et al., 2018a; Rienmuller and Borens, 2016; Figa et al., 2017; Dilisio et al., 2014). As in our case, culturenegative aspirations based on standard culture methods, with clear indications of infections based on synovial biomarkers, could indicate the presence of SGAB as the causative organism.

Culture of tissue samples is not just one of the oldest but also one of the most important methods in the diagnosis of IAI (Kamme et al., 1974; Kamme and Lindberg, 1981; Osmon et al., 2013; Parvizi et al., 2018). The method of collection and the number of samples collected can affect the culture results. To optimize the chance of culturing bacteria, antibiotic pause 2 weeks preoperatively and prophylactic antibiotics withheld until the samples are collected are often applied, although the value in SGAB IAI has not been investigated (Atkins et al., 1998). Multiple samples, also more than normal, may be required in SGAB due to a combination of low bacterial burden and uneven distribution of bacteria in the peri-implant tissues, as well as the risk of contamination in the processing of the samples (Atkins et al., 1998; McGoldrick et al., 2015). The current IDSA guidelines recommend at least three and optimally five or six samples with two positive cultures for a definitive diagnosis (Kamme and Lindberg, 1981; Osmon et al., 2013). However, a larger number of samples may be required for SGAB which have a lower rate of positive culture (Nodzo et al., 2016; McGoldrick et al., 2015; Akgun et al., 2018; Kheir et al., 2018). Kheir et al. (2018) calculated that to get two positive cultures the average number of samples required would be 10 for $C$. $a c$ nes, while $S$. aureus and coagulase-negative staphylococci (CoNS) would need just 3 and 4 samples, respectively (Kheir et al., 2018). But with the increase in number of samples comes a theoretic increase in false positive cultures due to contamination. The specific aspects in relation to SGAB IAI need further evaluation.

Since the introduction of sonication of removed implants in the 1990s by Tunney et al. (1998) and further improvements by Trampuz et al. (2007), microbial detection in sonication fluid culture has become widely adopted. Sonication is now incorporated in the IDSA and EBJIS guidelines for diagnosis of PJI (Osmon et al., 2013; McNally et al., 2021). Like other IAI-associated bacteria, SGAB have been shown to form biofilms on medical devices and sonication helps to dislodge these biofilms, making it easier to culture the bacteria (Achermann et al., 2014; Trampuz et al., 2007). Moreover, in patients with prior antibiotic therapy, sonication fluid specimens have shown to give more sensitive culture results than tissue specimens (Trampuz et al., 2007). For IAI caused by anaerobes, the culture of sonication fluid appears more sensitive than periprosthetic tissue samples (Portillo et al., 2014). Yet, several studies could not find any advantage of sonication fluid over tissue specimens in C. acnes IAI (Renz et al., 2018a; Akgun et al., 2020; Grosso et al., 2018). Conversely, the use of sonication fluid specimens was found to be inferior to the use of peri-implant tissue specimens in the detection of $C$. acnes in spinal implant infections (Sampedro et al., 2010).

The results from available studies regarding SGAB cultures are not easily comparable, as results depend on various factors, such as method of sample processing, choice of culture media, duration of incubation, and the cut-off chosen to determine significant microbial growth. For the processing of tissue samples, different methods of homogenization have been mentioned (Renz et al., 2018a; Figa et al., 2017; Zeller et al., 2018; Kheir et al., 2018; Grosso et al., 2018), and these could possibly affect the culture results too. Culture media used for the growth of anaerobic bacteria vary among studies. Anaerobic sheep blood agar (Renz et al., 2018a; Baumbach et al., 2018; Akgun et al., 2020; Grosso et al., 2018) and Columbia blood agar (Dilisio et al., 2014; Zeller et al., 2018) were the most commonly used solid media, while thioglycolate broth $(19,33,89,92,93)$ was the most commonly used liquid media. In addition, automated culture systems, like BACTEC or BacT/Alert, which are routinely used for blood culture and usually have the advantage of shorter time to positivity compared to conventional methods, were also used in some studies (Renz et al., 2018a; Portillo et al., 2014; Minassian et al., 2014; Sanabria et al., 2019). However, no consensus on the choice of media for SGAB exists, which makes evaluation of SGAB IAI a difficult task, as these IAIs 
can be potentially in an everyday clinical setting. The duration of cultivation is another important factor to consider, when it comes to detection of SGAB. Prolonged incubation of SGAB is usually required compared to other organisms. Previous studies have shown that while most bacteria causing orthopaedic infections were isolated within 1 to $5 \mathrm{~d}, C$. acnes can take anywhere from 6 to $14 \mathrm{~d}$ or longer to grow (Kheir et al., 2018; Kerneis et al., 2017; Nodzo et al., 2016; Garg et al., 2015). Incubation for $14 \mathrm{~d}$ seems to be the current standard for SGAB IAI (Renz et al., 2018a; Figa et al., 2017; Zeller et al., 2018; Baumbach et al., 2018; Nodzo et al., 2016; Kheir et al., 2018; Akgun et al., 2020; Portillo et al., 2014), though other studies have used $21 \mathrm{~d}$ (McGoldrick et al., 2015; Dilisio et al., 2014) and even 28 d (Lucas et al., 2016; Matsen et al., 2013). The need for prolonged incubation times must be considered against the increased risk of detection of contaminants with longer incubation periods. The number of bacterial colonies (colony forming units, CFU) that is cultivated is an important criterion to distinguish a true infection from possible contamination, and this topic is not evaluated for SGAB IAI. In sonication fluid different diagnostic CFU cut-offs $\left(20,50 \mathrm{CFU} \mathrm{mL}^{-1}\right.$, etc.) have been applied (Akgun et al., 2020; Grosso et al., 2018). The latest EBJIS definition of PJI (McNally et al., 2021) set the cut-off for confirmed infection to $>50$ and $>200 \mathrm{CFU} \mathrm{mL}^{-1}$ of any organism for uncentrifuged and centrifuged sonication fluid, respectively. Infection with SGAB was not differentiated in these guidelines (McNally et al., 2021).

\subsubsection{Culture-independent methods}

Culture-independent methods may have an important role in diagnosis of IAI, when other diagnostic tests are inconclusive, e.g. in the case of fastidious, slow-growing, or viable but non-culturable bacteria, and in patients with prior antibiotic therapy (McNally et al., 2021; Parvizi et al., 2018; Pasquaroli et al., 2013). Commonly used culture-independent methods include polymerase chain reaction (PCR)-based methods such as species-specific PCR, multiplex PCR, and 16S ribosomal RNA (rRNA) PCR and next-generation sequencing. The clinical downside to culture-independent methods is the lack of an antibiogram in the individual case.

The usefulness of PCR assays in IAI is debated. Sampedro et al. (2010) developed a species-specific $C$. acnes PCR assay, which amplifies a region of the $C$. acnes $16 \mathrm{~S}$ rRNA gene. The application of this $C$. acnes PCR assay to sonication fluid was compared with the results from sonication fluid culture. The PCR assay was able to detect only six of the nine C. acnes-positive cultures (Sampedro et al., 2010). The use of multiplex PCR assays that allow the simultaneous detection of multiple species of choice have yielded conflicting results. Morgenstern et al. (2018) used multiplex PCR and found that synovial fluid multiplex PCR was superior to culture for the detection of hip and knee PJI due to low virulent organisms (not SGAB) and gave earlier results ( $5 \mathrm{~h}$ versus up to $14 \mathrm{~d}$ )
(Morgenstern et al., 2018). However, other studies found that multiplex PCR of sonication fluid detected fewer pathogens compared to periprosthetic tissue culture and sonication fluid culture and missed several low virulent organisms, including SGAB (Cutibacterium spp. and Finegoldia magna) (Renz et al., 2017, 2018c). Bemer et al. (2014) found that the sensitivity of $16 \mathrm{~S}$ rRNA gene PCR assay was low for $C$. acnes, with only $11 \%$ of $C$. acnes and $72 \%$ of CoNS infections being detected by PCR, compared with $92 \%$ and $100 \%$ of S. aureus and streptococci, respectively (Bemer et al., 2014). The role of PCR based methods in SGAB IAI needs further evaluation. Amplicon next-generation sequencing (aNGS) or metagenomic next-generation sequencing (mNGS) have not been used, clinically, in the past for the diagnosis of IAI but is increasingly used in research on IAI. Its applicability is currently limited due to high costs and the need for extensive bioinformatics analyses but may have a future genuine clinical value in SGAB IAI. It involves DNA or RNA extraction, PCR amplification of target region (aNGS), enrichment of bacterial DNA or RNA (mNGS), library preparation, sequencing, and bioinformatics analysis (Chiu and Miller, 2019). The analysis can be targeted in aNGS, which involves the amplification of specific genomic regions of believed value or untargeted in mNGS (Chiu and Miller, 2019). The details and protocols of the individual steps can vary between individual laboratories, as these are currently at an experimental level. Next-generation sequencing analysis can be performed on any sample that yields sufficient nucleic acid (Chiu and Miller, 2019). Wang et al. (2020a) found in a study of 24 culture-negative PJIs that mNGS was able to detect rare pathogens and fastidious bacteria like Mycoplasma hominis, F. magna, and P. micra, which were clinically believed to be the causative organisms (Wang et al., 2020a). Wang et al. (2020b) found that the sensitivity of mNGS was significantly higher than the sensitivity of standard culture method, but not different to PCR assays (Wang et al., 2020b). Weaver et al. (2019) found in joint fluid from seven PJI cases that mNGS was able to detect more species including $C$. acnes and also was superior for the detection of polymicrobial infections compared to standard culture (Weaver et al., 2019). Namdari et al. (2019) found poor correlation between mNGS and culture in the detection of shoulder PJI (Namdari et al., 2019).

\section{Limitations of the review}

Most studies mentioned in this review investigated the diagnostic challenges and frequencies of PJI due to Cutibacterium spp. SGAB other than Cutibacterium species present with similar diagnostic challenges because of their similar clinical, imaging, laboratory, and microbiological findings, but only a limited number of studies focus on these SGAB in particular. 
Another limitation of the review is the underrepresentation of non-PJI IAI, since only a few studies focus on non-PJI IAI. This could be due to a lack of definitive diagnostic criteria for non-PJI IAI. But SGAB have been reported from IAI other than PJI, and their role in these infections should be investigated in more detail in future studies.

\section{Conclusion}

The presented case and review are in our opinion a clear example of the difficulties that SGAB IAIs present to orthopaedic surgeons, infectious disease specialists, and clinical microbiologists in performing best-practice of care for patients. Currently, a high degree of clinical suspicion based on up-to-date knowledge of SGAB is the only factor that can guide the clinician. Future research into diagnosing SGAB IAI, as specified above, is unquestionably warranted.

Data availability. No data sets were used in this article.

Author contributions. DSP prepared the article with contributions from all co-authors.

Competing interests. The contact author has declared that neither they nor their co-authors have any competing interests.

Disclaimer. Publisher's note: Copernicus Publications remains neutral with regard to jurisdictional claims in published maps and institutional affiliations.

Financial support. This research (project no. 30568) has been supported by "Fabrikant Vilhelm Pedersen og Hustrus Legat" by recommendation from the Novo Nordisk Foundation.

Review statement. This paper was edited by Parham Sendi and reviewed by three anonymous referees.

\section{References}

Achermann, Y., Sahin, F., Schwyzer, H. K., Kolling, C., Wust, J., and Vogt, M.: Characteristics and outcome of 16 periprosthetic shoulder joint infections, Infection, 41, 613620, https://doi.org/10.1007/s15010-012-0360-4, 2013.

Achermann, Y., Goldstein, E. J., Coenye, T., and Shirtliff, M. E.: Propionibacterium acnes: from commensal to opportunistic biofilm-associated implant pathogen, Clin. Microbiol. Rev., 27, 419-440, https://doi.org/10.1128/cmr.00092-13, 2014.

Achermann, Y., Liu, J., Zbinden, R., Zingg, P. O., Anagnostopoulos, A., Barnard, E., Sutter, R., Li, H., McDowell, A., and Zinkernagel, A. S.: Propionibacterium avidum: A Virulent Pathogen
Causing Hip Periprosthetic Joint Infection, Clin. Infect. Diseas., 66, 54-63, https://doi.org/10.1093/cid/cix665, 2017.

Akgun, D., Muller, M., Perka, C., and Winkler, T.: The serum level of C-reactive protein alone cannot be used for the diagnosis of prosthetic joint infections, especially in those caused by organisms of low virulence, Bone Joint J., 100B, 1482-1486, https://doi.org/10.1302/0301-620X.100B11.BJJ2018-0514.R1, 2018.

Akgun, D., Maziak, N., Plachel, F., Minkus, M., Scheibel, M., Perka, C., and Moroder, P.: Diagnostic Arthroscopy for Detection of Periprosthetic Infection in Painful Shoulder Arthroplasty, Arthroscopy, 35, 2571-2577, https://doi.org/10.1016/j.arthro.2019.03.058, 2019.

Akgun, D., Maziak, N., Plachel, F., Siegert, P., Minkus, M., Thiele, K., and Moroder, P.: The role of implant sonication in the diagnosis of periprosthetic shoulder infection, J. Shoulder Elbow Surg., 29, e222-e228, https://doi.org/10.1016/j.jse.2019.10.011, 2020.

Alijanipour, P., Bakhshi, H., and Parvizi, J.: Diagnosis of periprosthetic joint infection: the threshold for serological markers, Clin. Orthop. Relat. Res., 471, 3186-3195, https://doi.org/10.1007/s11999-013-3070-z, 2013.

Anagnostakos, K., Grzega, C., Sahan, I., Geipel, U., and Becker, S. L.: Occurrence of Rare Pathogens at the Site of Periprosthetic Hip and Knee Joint Infections: A Retrospective, Single-Center Study, Antibiotics (Basel), 10, 882, https://doi.org/10.3390/antibiotics10070882, 2021.

Atkins, B. L., Athanasou, N., Deeks, J. J., Crook, D. W. M., Simpson, H., Peto, T. E. A., McLardy-Smith, P., Berendt, A. R., and Group, T. O. C. S.: Prospective Evaluation of Criteria for Microbiological Diagnosis of Prosthetic-Joint Infection at Revision Arthroplasty, J. Clin. Microbiol., 36, 2932-2939, https://doi.org/10.1128/jcm.36.10.2932-2939.1998, 1998.

Aubin, G. G., Portillo, M. E., Trampuz, A., and Corvec, S.: Propionibacterium acnes, an emerging pathogen: from acne to implant-infections, from phylotype to resistance, Med. Mal. Infect., 44, 241-250, https://doi.org/10.1016/j.medmal.2014.02.004, 2014.

Bacle, G., Sikora, S. K., and Ek, E. T. H.: Propionibacterium Acnes Infection of a Metacarpophalangeal Joint Arthroplasty, J. Hand. Surg. Am., 42, 394.e391-394.e396, https://doi.org/10.1016/j.jhsa.2017.01.023, 2017.

Bartz, H., Nonnenmacher, C., Bollmann, C., Kuhl, M., Zimmermann, S., Heeg, K., and Mutters, R.: Micromonas (Peptostreptococcus) micros: unusual case of prosthetic joint infection associated with dental procedures, Int. J. Med. Microbiol., 294, 465470, 2005.

Baumbach, S. F., Prall, W. C., Scharpf, A. M., Hererich, V., Schmidt, M., Suedkamp, N. P., Stoehr, A., and Mayr, H. O.: Significant increase of pathogen detection rate by dry arthroscopic biopsies at suspected low-grade infection following total knee arthroplasty: a prospective observational study, Arch. Orthop. Trauma Surg., 138, 1583-1590, https://doi.org/10.1007/s00402018-3032-8, 2018.

Bemer, P., Plouzeau, C., Tande, D., Leger, J., Giraudeau, B., Valentin, A. S., Jolivet-Gougeon, A., Vincent, P., Corvec, S., Gibaud, S., Juvin, M. E., Hery-Arnaud, G., Lemarie, C., Kempf, M., Bret, L., Quentin, R., Coffre, C., de Pinieux, G., Bernard, L., Burucoa, C., and Centre de Reference des Infections Osteo-articulaires du Grand Ouest Study, T.: Evalua- 
tion of 16S rRNA gene PCR sensitivity and specificity for diagnosis of prosthetic joint infection: a prospective multicenter cross-sectional study, J. Clin. Microbiol., 52, 3583-3589, https://doi.org/10.1128/JCM.01459-14, 2014.

Bjerke-Kroll, B. T., Christ, A. B., McLawhorn, A. S., Sculco, P. K., Jules-Elysee, K. M., and Sculco, T. P.: Periprosthetic joint infections treated with two-stage revision over 14 years: an evolving microbiology profile, J. Arthroplast., 29, 877-882, https://doi.org/10.1016/j.arth.2013.09.053, 2014.

Bonanzinga, T., Zahar, A., Dutsch, M., Lausmann, C., Kendoff, D., and Gehrke, T.: How Reliable Is the Alpha-defensin Immunoassay Test for Diagnosing Periprosthetic Joint Infection? A Prospective Study, Clin. Orthop. Relat. Res., 475, 408-415, https://doi.org/10.1007/s11999-016-4906-0, 2017.

Boni, L., Kuster, S. P., Bartik, B., Zbinden, R., Zingg, P. O., andm Achermann, Y.: Association of Cutibacterium avidum Colonization in the Groin With Obesity: A Potential Risk Factor for Hip Periprosthetic Joint Infection, Clin. Infect. Dis., 67, 1878-1882, https://doi.org/10.1093/cid/ciy379, 2018.

Bossard, D. A., Ledergerber, B., Zingg, P. O., Gerber, C., Zinkernagel, A. S., Zbinden, R., and Achermann, Y.: Optimal Length of Cultivation Time for Isolation of Propionibacterium acnes in Suspected Bone and Joint Infections Is More than 7 Days, J. Clin. Microbiol., 54, 3043-3049, https://doi.org/10.1128/jcm.01435$16,2016$.

Both, A., Klatte, T. O., Lubke, A., Buttner, H., Hartel, M. J., Grossterlinden, L. G., and Rohde, H.: Growth of Cutibacterium acnes is common on osteosynthesis material of the shoulder in patients without signs of infection, Acta Orthop., 89, 580-584, https://doi.org/10.1080/17453674.2018.1489095, 2018.

Brüggemann, H., Poehlein, A., Brzuszkiewicz, E., Scavenius, C., Enghild, J. J., Al-Zeer, M. A., Brinkmann, V., Jensen, A., and Soderquist, B.: Staphylococcus saccharolyticus Isolated From Blood Cultures and Prosthetic Joint Infections Exhibits Excessive Genome Decay, Front. Microbiol., 10, 478, https://doi.org/10.3389/fmicb.2019.00478, 2019.

Butler-Wu, S. M., Burns, E. M., Pottinger, P. S., Magaret, A. S., Rakeman, J. L., Matsen III, F. A., and Cookson, B. T.: Optimization of periprosthetic culture for diagnosis of Propionibacterium acnes prosthetic joint infection, J. Clin. Microbiol., 49, 24902495, https://doi.org/10.1128/JCM.00450-11, 2011.

Chiu, C. Y. and Miller, S. A.: Clinical metagenomics, Nat. Rev. Genet., 20, 341-355, https://doi.org/10.1038/s41576-019-01137, 2019.

Corona, P. S., Haddad, S., Andres, J., Gonzalez-Lopez, J. J., Amat, C., and Flores, X.: Case report: first report of a prosthetic joint infection caused by Facklamia hominis, Diagn. Microbiol. Infect. Dis., 80, 338-340, https://doi.org/10.1016/j.diagmicrobio.2014.08.008, 2014.

Deirmengian, C., Hallab, N., Tarabishy, A., Della Valle, C., Jacobs, J. J., Lonner, J., and Booth Jr., R. E.: Synovial fluid biomarkers for periprosthetic infection, Clin. Orthop. Relat. Res., 468, 20172023, https://doi.org/10.1007/s11999-010-1298-4, 2010.

Deirmengian, C., Kardos, K., Kilmartin, P., Cameron, A., Schiller, K., and Parvizi, J.: Diagnosing periprosthetic joint infection: has the era of the biomarker arrived?, Clin. Orthop. Relat. Res., 472, 3254-3262, https://doi.org/10.1007/s11999-014-3543-8, 2014.

Di Benedetto, P., Dalla Vecchia, G., Dante, F., Gisonni, R., Cainero, V., and Causero, A.: Leukocyte esterase strip test as a reliable intraoperative PJIs biomarker. Our experience, Acta Biomed., 90, 43-47, https://doi.org/10.23750/abm.v90i12-S.8993, 2019.

Dilisio, M. F., Miller, L. R., Warner, J. J., and Higgins, L. D.: Arthroscopic tissue culture for the evaluation of periprosthetic shoulder infection, J. Bone Joint Surg. Am., 96, 1952-1958, https://doi.org/10.2106/jbjs.M.01512, 2014.

Dodson, C. C., Craig, E. V., Cordasco, F. A., Dines, D. M., Dines, J. S., Dicarlo, E., Brause, B. D., and Warren, R. F.: Propionibacterium acnes infection after shoulder arthroplasty: a diagnostic challenge, J. Shoulder Elbow Surg., 19, 303-307, https://doi.org/10.1016/j.jse.2009.07.065, 2010.

Drago, L., De Vecchi, E., Bortolin, M., Zagra, L., Romano, C. L., and Cappelletti, L.: Epidemiology and Antibiotic Resistance of Late Prosthetic Knee and Hip Infections, J. Arthroplast., 32, 2496-2500, https://doi.org/10.1016/j.arth.2017.03.005, 2017.

Evans, C. A., Mattern, K. L., and Hallam, S. L.: Isolation and identification of Peptococcus saccharolyticus from human skin, J. Clin. Microbiol., 7, 261-264, 1978.

Falstie-Jensen, T., Daugaard, H., Soballe, K., Ovesen, J., Arveschoug, A. K., and Lange, J.: Labeled white blood cell/bone marrow single-photon emission computed tomography with computed tomography fails in diagnosing chronic periprosthetic shoulder joint infection, J. Shoulder Elbow Surg., 28, 1040 1048, https://doi.org/10.1016/j.jse.2018.10.024, 2019a.

Falstie-Jensen, T., Daugaard, H., Soballe, K., Ovesen, J., Arveschoug, A. K., Lange, J., and Rosa study-group: Labeled white blood cell/bone marrow single-photon emission computed tomography with computed tomography fails in diagnosing chronic periprosthetic shoulder joint infection, J. Shoulder Elbow Surg., 28, 1040-1048, https://doi.org/10.1016/j.jse.2018.10.024, 2019b.

Falstie-Jensen, T., Lange, J., Daugaard, H., Vendelbo, M. H., Sørensen, A. K., Zerahn, B., Ovesen, J., Søballe, K., Gormsen, L. C., Olsen, B. S., Johanssen, H. V. S., Elmengaard, B., Thillemann, T. M., Bolvig, L., and on behalf of the Rosa study-group: ${ }^{18} \mathrm{~F}$ FDG-PET/CT has poor diagnostic accuracy in diagnosing shoulder PJI, Eur. J. Nucl. Med. Molec. Imag., 46, 2013-2022, https://doi.org/10.1007/s00259-019-04381-w, 2019c.

Falstie-Jensen, T., Lange, J., Daugaard, H., Sørensen, A. K. B., Ovesen, J., and Søballe, K.: Unexpected positive cultures after revision shoulder arthroplasty: does it affect outcome?, J. Shoulder Elbow Surg., 30, 1299-1308, https://doi.org/10.1016/j.jse.2020.12.014, 2021.

Figa, R., Muneton, D., Gomez, L., Matamala, A., Lung, M., Cuchi, E., and Corona, P. S.: Periprosthetic joint infection by Propionibacterium acnes: Clinical differences between monomicrobial versus polymicrobial infection, Anaerobe, 44, 143-149, https://doi.org/10.1016/j.anaerobe.2017.03.008, 2017.

Fischbacher, A. and Borens, O.: Prosthetic-joint Infections: Mortality Over The Last 10 Years, J. Bone Joint Infect., 4, 198-202, https://doi.org/10.7150/jbji.35428, 2019.

Foruria, A. M., Fox, T. J., Sperling, J. W., and Cofield, R. H.: Clinical meaning of unexpected positive cultures (UPC) in revision shoulder arthroplasty, J. Shoulder Elbow Surg., 22, 620-627, https://doi.org/10.1016/j.jse.2012.07.017, 2013.

Frangiamore, S. J., Saleh, A., Kovac, M. F., Grosso, M. J., Zhang, X., Bauer, T. W., Daly, T. M., Ricchetti, E. T., and Iannotti, J. P.: Synovial fluid interleukin-6 as a predictor of peripros- 
thetic shoulder infection, J. Bone Joint Surg. Am., 97, 63-70, https://doi.org/10.2106/jbjs.N.00104, 2015.

Garg, S., LaGreca, J., Hotchkiss, M., and Erickson, M.: Management of late $(>1 \mathrm{y})$ deep infection after spinal fusion: a retrospective cohort study, J. Pediatr. Orthop., 35, 266-270, https://doi.org/10.1097/bpo.0000000000000252, 2015.

Gausden, E. B., Villa, J., Warner, S. J., Redko, M., Pearle, A., Miller, A., Henry, M., Lorich, D. G., Helfet, D. L., and Wellman, D. S.: Nonunion After Clavicle Osteosynthesis: High Incidence of Propionibacterium acnes, J. Orthop. Trauma, 31, 229235, https://doi.org/10.1097/bot.0000000000000770, 2017.

Gehrke, T., Lausmann, C., Citak, M., Bonanzinga, T., Frommelt, L., and Zahar, A.: The Accuracy of the Alpha Defensin Lateral Flow Device for Diagnosis of Periprosthetic Joint Infection: Comparison with a Gold Standard, J. Bone Joint Surg. Am., 100, 42-48, https://doi.org/10.2106/JBJS.16.01522, 2018.

Gemmel, F., Van den Wyngaert, H., Love, C., Welling, M. M., Gemmel, P., and Palestro, C. J.: Prosthetic joint infections: radionuclide state-of-the-art imaging, Eur. J. Nucl. Med. Molec. Imag., 39, 892-909, https://doi.org/10.1007/s00259-012-2062-7, 2012.

Grosso, M. J., Frangiamore, S. J., Ricchetti, E. T., Bauer, T. W., and Iannotti, J. P.: Sensitivity of frozen section histology for identifying Propionibacterium acnes infections in revision shoulder arthroplasty, J. Bone Joint Surg. Am., 96, 442-447, https://doi.org/10.2106/jbjs.M.00258, 2014a.

Grosso, M. J., Frangiamore, S. J., Saleh, A., Kovac, M. F., Hayashi, R., Ricchetti, E. T., Bauer, T. W., and Iannotti, J. P.: Poor utility of serum interleukin-6 levels to predict indolent periprosthetic shoulder infections, J. Shoulder Elbow Surg., 23, 1277-1281, https://doi.org/10.1016/j.jse.2013.12.023, 2014b.

Grosso, M. J., Frangiamore, S. J., Yakubek, G., Bauer, T. W., Iannotti, J. P., and Ricchetti, E. T.: Performance of implant sonication culture for the diagnosis of periprosthetic shoulder infection, J. Shoulder Elbow Surg., 27, 211-216, https://doi.org/10.1016/j.jse.2017.08.008, 2018.

Hou, C., Gupta, A., Chen, M., and Matsen III, F. A.: How do revised shoulders that are culture positive for Propionibacterium differ from those that are not?, J. Shoulder Elbow Surg., 24, 14271432, https://doi.org/10.1016/j.jse.2015.01.003, 2015.

Hsu, J. E., Neradilek, M. B., Russ, S. M., and Matsen III, F. A.: Preoperative skin cultures are predictive of Propionibacterium load in deep cultures obtained at revision shoulder arthroplasty, J. Shoulder Elbow Surg., 27, 765-770, https://doi.org/10.1016/j.jse.2018.01.021, 2018.

Huang, J., Zhang, Y., Wang, Z., Dong, Y., Zhao, Y., Zheng, J., Lian, H., and Jin, Y.: The serum level of D-Dimer is not suitable for distinguishing between prosthetic joint infection and aseptic loosening, J. Orthop. Surg. Res., 14, 407, https://doi.org/10.1186/s13018-019-1461-x, 2019.

Huang, Z., Zhang, C., Li, W., Fang, X., Wang, Q., Xing, L., Li, Y., Nie, X., Yang, B., and Zhang, W.: Metagenomic next-generation sequencing contribution in identifying prosthetic joint infection due to Parvimonas micra: a case report, J. Bone Joint Infect., 4, 50-55, https://doi.org/10.7150/jbji.30615, 2019.

Hudek, R., Sommer, F., Kerwat, M., Abdelkawi, A. F., Loos, F., and Gohlke, F.: Propionibacterium acnes in shoulder surgery: true infection, contamination, or commensal of the deep tissue?, J. Shoulder Elbow Surg., 23, 1763-1771, https://doi.org/10.1016/j.jse.2014.05.024, 2014.
Hudek, R., Brobeil, A., Brüggemann, H., Sommer, F., Gattenlöhner, S., and Gohlke, F.: Cutibacterium acnes is an intracellular and intra-articular commensal of the human shoulder joint, J. Shoulder Elbow Surg., 30, 16-26, https://doi.org/10.1016/j.jse.2020.04.020, 2021.

Jacobs, A. M., Van Hooff, M. L., Meis, J. F., Vos, F., and Goosen, J. H.: Treatment of prosthetic joint infections due to Propionibacterium. Similar results in 60 patients treated with and without rifampicin, Acta Orthop., 87, 60-66, https://doi.org/10.3109/17453674.2015.1094613, 2016.

Kamme, C. and Lindberg, L.: Aerobic and anaerobic bacteria in deep infections after total hip arthroplasty: differential diagnosis between infectious and non-infectious loosening, Clin. Orthop. Relat. Res., 154, 201-207, 1981.

Kamme, C., Lidgren, L., Lindberg, L., and Mardh, P. A.: Anaerobic bacteria in late infections after total hip arthroplasty, Scand. J. Infect. Dis., 6, 161-165, https://doi.org/10.3109/inf.1974.6.issue2.10, 1974.

Kerneis, S., Leprince, C., Archambeau, D., Eyrolle, L., Leclerc, P., Poupet, H., Loubinoux, J., Gauzit, R., Salmon, D., Launay, O., Poyart, C., Anract, P., and Morand, P. C.: Impact of Sequential Culture Results on Diagnosis and De-Escalation of the Antibiotic Regimen in Joint and Bone Infections, Surg. Infect. (Larchmt), 18, 910-914, https://doi.org/10.1089/sur.2017.106, 2017.

Kheir, M. M., Tan, T. L., Ackerman, C. T., Modi, R., Foltz, C., and Parvizi, J.: Culturing Periprosthetic Joint Infection: Number of Samples, Growth Duration, and Organisms, J. Arthroplast., 33, 3531-3536, https://doi.org/10.1016/j.arth.2018.06.018, 2018.

Lange, J., Troelsen, A., Solgaard, S., Otte, K. S., Jensen, N. K., Soballe, K., and CORIHA research group: Cementless OneStage Revision in Chronic Periprosthetic Hip Joint Infection. Ninety-One Percent Infection Free Survival in 56 Patients at Minimum 2-Year Follow-Up, J. Arthroplast., 33, 1160-1165, https://doi.org/10.1016/j.arth.2017.11.024, 2018.

Larsen, L. H., Lange, J., Xu, Y., and Schonheyder, H. C.: Optimizing culture methods for diagnosis of prosthetic joint infections: a summary of modifications and improvements reported since 1995, J. Med. Microbiol., 61, 309-316, https://doi.org/10.1099/jmm.0.035303-0, 2012.

Lebowitz, D., Kressmann, B., Gjoni, S., Zenelaj, B., Grosgurin, O., Marti, C., Zingg, M., and Uckay, I.: Clinical features of anaerobic orthopaedic infections, Infect. Dis. (Lond.), 49, 137-140, https://doi.org/10.1080/23744235.2016.1225979, 2017.

Levy, P. Y., Fenollar, F., Stein, A., Borrione, F., and Raoult, D.: Finegoldia magna: a forgotten pathogen in prosthetic joint infection rediscovered by molecular biology, Clin. Infect. Dis., 49, 12441247, https://doi.org/10.1086/605672, 2009.

Lima, A. L., Oliveira, P. R., Carvalho, V. C., Saconi, E. S., Cabrita, H. B., and Rodrigues, M. B.: Periprosthetic joint infections, Interdiscip. Perspect. Infect. Dis., 2013, 542796, https://doi.org/10.1155/2013/542796, 2013.

Lin, Z. X., Steed, L. L., Marculescu, C. E., Slone, H. S., and Woolf, S. K.: Cutibacterium acnes Infection in Orthopedics: Microbiology, Clinical Findings, Diagnostic Strategies, and Management, Orthopedics, 43, 52-61, https://doi.org/10.3928/0147744720191213-02, 2020.

Lucas, R. M., Hsu, J. E., Whitney, I. J., Wasserburger, J., and Matsen III, F. A.: Loose glenoid components in revision shoulder arthroplasty: is there an association with pos- 
itive cultures?, J. Shoulder Elbow Surg., 25, 1371-1375, https://doi.org/10.1016/j.jse.2015.12.026, 2016.

Lutz, J. A., Otten, P., and Maestretti, G.: Late infections after dynamic stabilization of the lumbar spine with Dynesys, Eur. Spine J., 21, 2573-2579, https://doi.org/10.1007/s00586-012-2366-0, 2012.

Marmor, S., Bauer, T., Desplaces, N., Heym, B., Roux, A. L., Sol, O., Roge, J., Mahe, F., Desire, L., Aegerter, P., Ghout, I., Ropers, J., Gaillard, J. L., and Rottman, M.: Multiplex Antibody Detection for Noninvasive Genus-Level Diagnosis of Prosthetic Joint Infection, J. Clin. Microbiol., 54, 1065-1073, https://doi.org/10.1128/jcm.02885-15, 2016.

Maroto Piñeiro, F., Álvarez Otero, J., Lamas Ferreiro, J. L., Sanjurjo Rivo, A. B., Pintado García, A., and de la Fuente Aguado, J.: Late onset prosthetic joint infection caused by Parvimonas micra, Anaerobe, 71, 102414, https://doi.org/10.1016/j.anaerobe.2021.102414, 2021.

Matsen III, F. A., 3rd, Butler-Wu, S., Carofino, B. C., Jette, J. L., Bertelsen, A., and Bumgarner, R.: Origin of propionibacterium in surgical wounds and evidence-based approach for culturing propionibacterium from surgical sites, J. Bone Joint Surg. Am., 95, 1811-1817, https://doi.org/10.2106/JBJS.L.01733, 2013.

Matsen III, F. A., Russ, S. M., Bertelsen, A., Butler-Wu, S., and Pottinger, P. S.: Propionibacterium can be isolated from deep cultures obtained at primary arthroplasty despite intravenous antimicrobial prophylaxis, J. Shoulder Elbow Surg., 24, 844-847, https://doi.org/10.1016/j.jse.2014.10.016, 2015.

Matsen III, F. A., Whitson, A. J., Pottinger, P. S., Neradilek, M. B., and Hsu, J. E.: Cutaneous microbiology of patients having primary shoulder arthroplasty, J. Shoulder Elbow Surg., 29, 16711680, https://doi.org/10.1016/j.jse.2019.12.031, 2020.

McGoldrick, E., McElvany, M. D., Butler-Wu, S., Pottinger, P. S., and Matsen III, F. A.: Substantial cultures of Propionibacterium can be found in apparently aseptic shoulders revised three years or more after the index arthroplasty, J. Shoulder Elbow Surg., 24, 31-35, https://doi.org/10.1016/j.jse.2014.05.008, 2015.

McNally, M., Sousa, R., Wouthuyzen-Bakker, M., Chen, A. F., Soriano, A., Vogely, H. C., Clauss, M., Higuera, C. A., and Trebše, R.: The EBJIS definition of periprosthetic joint infection, Bone Joint J., 103-b, 18-25, https://doi.org/10.1302/0301620x.103b1.Bjj-2020-1381.R1, 2021.

Minassian, A. M., Newnham, R., Kalimeris, E., Bejon, P., Atkins, B. L., and Bowler, I. C.: Use of an automated blood culture system (BD BACTEC) for diagnosis of prosthetic joint infections: easy and fast, BMC Infect. Dis., 14, 233, https://doi.org/10.1186/1471-2334-14-233, 2014.

Morgenstern, C., Cabric, S., Perka, C., Trampuz, A., and Renz, N.: Synovial fluid multiplex PCR is superior to culture for detection of low-virulent pathogens causing periprosthetic joint infection, Diagn. Microbiol. Infect. Dis., 90, 115-119, https://doi.org/10.1016/j.diagmicrobio.2017.10.016, 2018.

Namdari, S., Nicholson, T., Abboud, J., Lazarus, M., Ramsey, M. L., Williams, G., and Parvizi, J.: Comparative study of cultures and next-generation sequencing in the diagnosis of shoulder prosthetic joint infections, J. Shoulder Elbow Surg., 28, 1-8, https://doi.org/10.1016/j.jse.2018.08.048, 2019.

Nodzo, S. R., Westrich, G. H., Henry, M. W., and Miller, A. O.: Clinical Analysis of Propionibacterium acnes Infection Af- ter Total Knee Arthroplasty, J. Arthroplast., 31, 1986-1989, https://doi.org/10.1016/j.arth.2016.02.025, 2016.

Nystrom, L. M., Wyatt, C. M., and Noiseux, N. O.: Arthroplasty infection by Priopionibacterium granulosum treated with reimplantation despite ongoing purulentappearing fluid collection, J. Arthroplast., 28, 195-198, https://doi.org/10.1016/j.arth.2012.03.004, 2013.

Omar, M., Ettinger, M., Reichling, M., Petri, M., Guenther, D., Gehrke, T., Krettek, C., and Mommsen, P.: Synovial Creactive protein as a marker for chronic periprosthetic infection in total hip arthroplasty, Bone Joint J., 97-b, 173-176, https://doi.org/10.1302/0301-620x.97b2.34550, 2015.

Osmon, D. R., Berbari, E. F., Berendt, A. R., Lew, D., Zimmerli, W., Steckelberg, J. M., Rao, N., Hanssen, A., Wilson, W. R., and Infectious Diseases Society of America: Executive summary: diagnosis and management of prosthetic joint infection: clinical practice guidelines by the Infectious Diseases Society of America, Clin. Infect. Dis., 56, 1-10, https://doi.org/10.1093/cid/cis966, 2013.

Ottink, K. D., Strahm, C., Muller-Kobold, A., Sendi, P., and Wouthuyzen-Bakker, M.: Factors to Consider When Assessing the Diagnostic Accuracy of Synovial Leukocyte Count in Periprosthetic Joint Infection, J. Bone Joint Infect., 4, 167-173, https://doi.org/10.7150/jbji.34854, 2019.

Padegimas, E. M., Lawrence, C., Narzikul, A. C., Zmistowski, B. M., Abboud, J. A., Williams, G. R., and Namdari, S.: Future surgery after revision shoulder arthroplasty: the impact of unexpected positive cultures, J. Shoulder Elbow Surg., 26, 975-981, https://doi.org/10.1016/j.jse.2016.10.023, 2017.

Parvizi, J., Zmistowski, B., Berbari, E. F., Bauer, T. W., Springer, B. D., Della Valle, C. J., Garvin, K. L., Mont, M. A., Wongworawat, M. D., and Zalavras, C. G.: New definition for periprosthetic joint infection: from the Workgroup of the Musculoskeletal Infection Society, Clin. Orthop. Relat. Res., 469, 2992-2994, https://doi.org/10.1007/s11999-011-2102-9, 2011.

Parvizi, J., Tan, T. L., Goswami, K., Higuera, C., Della Valle, C., Chen, A. F., and Shohat, N.: The 2018 Definition of Periprosthetic Hip and Knee Infection: An EvidenceBased and Validated Criteria, J. Arthroplast., 33, 1309-1314, https://doi.org/10.1016/j.arth.2018.02.078, 2018.

Pasquaroli, S., Zandri, G., Vignaroli, C., Vuotto, C., Donelli, G., and Biavasco, F.: Antibiotic pressure can induce the viable but non-culturable state in Staphylococcus aureus growing in biofilms, J. Antimicrob. Chemoth., 68, 1812-1817, https://doi.org/10.1093/jac/dkt086, 2013.

Plaass, C., Hasler, C. C., Heininger, U., and Studer, D.: Bacterial colonization of VEPTR implants under repeated expansions in children with severe early onset spinal deformities, Eur. Spine J., 25, 549-556, https://doi.org/10.1007/s00586-015-4003-1, 2016.

Portillo, M. E., Corvec, S., Borens, O., and Trampuz, A.: Propionibacterium acnes: an underestimated pathogen in implantassociated infections, Biomed. Res. Int., 2013, 804391, https://doi.org/10.1155/2013/804391, 2013.

Portillo, M. E., Salvado, M., Alier, A., Martinez, S., Sorli, L., Horcajada, J. P., and Puig, L.: Advantages of sonication fluid culture for the diagnosis of prosthetic joint infection, J. Infect., 69, 3541, https://doi.org/10.1016/j.jinf.2014.03.002, 2014.

Pottinger, P., Butler-Wu, S., Neradilek, M. B., Merritt, A., Bertelsen, A., Jette, J. L., Warme, W. J., and Matsen III, F. 
A.: Prognostic factors for bacterial cultures positive for Propionibacterium acnes and other organisms in a large series of revision shoulder arthroplasties performed for stiffness, pain, or loosening, J. Bone Joint Surg. Am., 94, 2075-2083, https://doi.org/10.2106/jbjs.K.00861, 2012.

Puchner, S. E., Doring, K., Staats, K., Bohler, C., Lass, R., Hirschl, A. M., Presterl, E., Windhager, R., and Holinka, J.: Sonication culture improves microbiological diagnosis of modular megaprostheses, J. Orthop. Res., 35, 1383-1387, https://doi.org/10.1002/jor.23406, 2017.

Pumberger, M., Burger, J., Strube, P., Akgun, D., and Putzier, M.: Unexpected positive cultures in presumed aseptic revision spine surgery using sonication, Bone Joint J., 101b, 621-624, https://doi.org/10.1302/0301-620x.101b5.Bjj-20181168.R1, 2019.

Randall, D., Jee, Y., Vanood, A., and Mayo, D.: Atypical Presentation of Periprosthetic Joint Infection After Total Knee Arthroplasty due to Parvimonas micra, Arthroplast. Today, 6, 901-905, https://doi.org/10.1016/j.artd.2020.09.021, 2020.

Renz, N., Feihl, S., Cabric, S., and Trampuz, A.: Performance of automated multiplex PCR using sonication fluid for diagnosis of periprosthetic joint infection: a prospective cohort, Infection, 45, 877-884, https://doi.org/10.1007/s15010-017-1073-5, 2017.

Renz, N., Mudrovcic, S., Perka, C., and Trampuz, A.: Orthopedic implant-associated infections caused by Cutibacterium spp. - A remaining diagnostic challenge, PLoS One, 13, e0202639, https://doi.org/10.1371/journal.pone.0202639, 2018a.

Renz, N., Yermak, K., Perka, C., and Trampuz, A.: Alpha Defensin Lateral Flow Test for Diagnosis of Periprosthetic Joint Infection: Not a Screening but a Confirmatory Test, J. Bone Joint Surg. Am., 100, 742-750, https://doi.org/10.2106/JBJS.17.01005, 2018 b.

Renz, N., Cabric, S., Morgenstern, C., Schuetz, M. A., and Trampuz, A.: Value of PCR in sonication fluid for the diagnosis of orthopedic hardware-associated infections: Has the molecular era arrived?, Injury, 49, 806-811, https://doi.org/10.1016/j.injury.2018.02.018, 2018c.

Richards, J., Inacio, M. C., Beckett, M., Navarro, R. A., Singh, A., Dillon, M. T., Sodl, J. F., and Yian, E. H.: Patient and procedure-specific risk factors for deep infection after primary shoulder arthroplasty, Clin. Orthop. Relat. Res., 472, 2809-2815, https://doi.org/10.1007/s11999-014-3696-5, 2014.

Rieber, H., Frontzek, A., Bell, A., and Frommelt, L.: Robinsoniella peoriensis, originally isolated from swine manure, and early periprosthetic hip infection: Case report and review of the literature, Anaerobe, 42, 33-36, https://doi.org/10.1016/j.anaerobe.2016.07.005, 2016.

Rieber, H., Frontzek, A., Jerosch, J., Alefeld, M., Strohecker, T., Ulatowski, M., Morawietz, T., Hinsenkamp, S., Bell, A., Kucukkoylu, D., and Frommelt, L.: Periprosthetic joint infection caused by anaerobes. Retrospective analysis reveals no need for prolonged cultivation time if sensitive supplemented growth media are used, Anaerobe, 50, 12-18, https://doi.org/10.1016/j.anaerobe.2018.01.009, 2018.

Rieber, H., Frontzek, A., and Schmitt, H.: Slackia exigua, an anaerobic Gram-positive rod and part of human oral microbiota associated with periprosthetic joint infection of the hip. First case and review of the literature, Anaerobe, 56, 130-132, https://doi.org/10.1016/j.anaerobe.2019.02.015, 2019.
Rieber, H., Frontzek, A., Alefeld, M., Heinrich, S., Barden, B., Jerosch, J., Breil-Wirth, A., Schmitt, H., Ulatowski, M., Gotz, S., Mullahi, A., Fischer, M., Weber, R., Pfander, D., Sakkal, A., Kukuk, P., and Bell, A.: Sonicate fluid inoculated into blood culture bottles does not improve diagnosis of periprosthetic joint infection caused by anaerobes. A retrospective analysis, Anaerobe, 62, 102152, https://doi.org/10.1016/j.anaerobe.2020.102152, 2020.

Rienmuller, A. and Borens, O.: Propionibacterium prosthetic joint infection: experience from a retrospective database analysis, Eur. J. Orthop. Surg. Traumatol., 26, 429-434, https://doi.org/10.1007/s00590-016-1766-y, 2016.

Saleh, A., George, J., Faour, M., Klika, A. K., and Higuera, C. A.: Serum biomarkers in periprosthetic joint infections, Bone Joint Res., 7, 85-93, https://doi.org/10.1302/2046-3758.71.Bjr-20170323, 2018.

Sampedro, M. F., Piper, K. E., McDowell, A., Patrick, S., Mandrekar, J. N., Rouse, M. S., Steckelberg, J. M., and Patel, R.: Species of Propionibacterium and Propionibacterium acnes phylotypes associated with orthopedic implants, Diagn. Microbiol. Infect. Dis., 64, 138-145, https://doi.org/10.1016/j.diagmicrobio.2009.01.024, 2009.

Sampedro, M. F., Huddleston, P. M., Piper, K. E., Karau, M. J., Dekutoski, M. B., Yaszemski, M. J., Currier, B. L., Mandrekar, J. N., Osmon, D. R., McDowell, A., Patrick, S., Steckelberg, J. M., and Patel, R.: A biofilm approach to detect bacteria on removed spinal implants, Spine (Phila Pa 1976), 35, 1218-1224, https://doi.org/10.1097/BRS.0b013e3181c3b2f3, 2010.

Sanabria, A., Rokeberg, M. E. O., Johannessen, M., Sollid, J. E., Simonsen, G. S., and Hanssen, A. M.: Culturing periprosthetic tissue in BacT/Alert(R) Virtuo blood culture system leads to improved and faster detection of prosthetic joint infections, BMC Infect. Dis., 19, 607, https://doi.org/10.1186/s12879-019-4206$\mathrm{x}, 2019$.

Sevelda, F. and Fink, B.: One-stage exchange of septic shoulder arthroplasty following a standardized treatment algorithm, J. Shoulder Elbow Surg., 27, 2175-2182, https://doi.org/10.1016/j.jse.2018.06.004, 2018.

Shah, N. B., Tande, A. J., Patel, R., and Berbari, E. F.: Anaerobic prosthetic joint infection, Anaerobe, 36, 1-8, https://doi.org/10.1016/j.anaerobe.2015.08.003, 2015.

Shahi, A., Kheir, M. M., Tarabichi, M., Hosseinzadeh, H. R. S., Tan, T. L., and Parvizi, J.: Serum D-Dimer Test Is Promising for the Diagnosis of Periprosthetic Joint Infection and Timing of Reimplantation, J. Bone Joint Surg. Am., 99, 1419-1427, https://doi.org/10.2106/JBJS.16.01395, 2017.

Singh, J. A., Sperling, J. W., Schleck, C., Harmsen, W. S., and Cofield, R. H.: Periprosthetic infections after total shoulder arthroplasty: a 33-year perspective, J. Shoulder Elbow Surg., 21, 1534-1541, https://doi.org/10.1016/j.jse.2012.01.006, 2012.

Söderquist, B., Bjorklund, S., Hellmark, B., Jensen, A., and Brüggemann, H.: Finegoldia magna Isolated from Orthopedic Joint Implant-Associated Infections, J. Clin. Microbiol., 55, 32833291, https://doi.org/10.1128/jcm.00866-17, 2017.

Söderquist, B., Afshar, M., Poehlein, A., and Brüggemann, H.: Staphylococcus saccharolyticus Associated with Prosthetic Joint Infections: Clinical Features and Genomic Characteristics, Pathogens, 10, 397, https://doi.org/10.3390/pathogens10040397, 2021. 
Stoll, T., Stucki, G., Bruhlmann, P., Vogt, M., Gschwend, N., and Michel, B. A.: Infection of a total knee joint prosthesis by peptostreptococcus micros and propionibacterium acnes in an elderly RA patient: implant salvage with longterm antibiotics and needle aspiration/irrigation, Clin. Rheumatol., 15, 399-402, https://doi.org/10.1007/bf02230366, 1996.

Tande, A. J. and Patel, R.: Prosthetic joint infection, Clin. Microbiol. Rev., 27, 302-345, https://doi.org/10.1128/CMR.00111-13, 2014.

Tarabichi, M., Shohat, N., Goswami, K., Alvand, A., Silibovsky, R., Belden, K., and Parvizi, J.: Diagnosis of Periprosthetic Joint Infection: The Potential of Next-Generation Sequencing, J. Bone Joint Surg., 100, 147-154, https://doi.org/10.2106/jbjs.17.00434, 2018.

Tetreault, M. W., Wetters, N. G., Moric, M., Gross, C. E., and Della Valle, C. J.: Is synovial C-reactive protein a useful marker for periprosthetic joint infection?, Clin. Orthop. Relat. Res., 472, 3997-4003, https://doi.org/10.1007/s11999-014-3828-y, 2014.

Trampuz, A., Piper, K. E., Jacobson, M. J., Hanssen, A. D., Unni, K. K., Osmon, D. R., Mandrekar, J. N., Cockerill, F. R., Steckelberg, J. M., Greenleaf, J. F., and Patel, R.: Sonication of removed hip and knee prostheses for diagnosis of infection, N. Engl. J. Med., 357, 654-663, https://doi.org/10.1056/NEJMoa061588, 2007.

Triffault-Fillit, C., Ferry, T., Laurent, F., Pradat, P., Dupieux, C., Conrad, A., Becker, A., Lustig, S., Fessy, M. H., Chidiac, C., and Valour, F.: Microbiologic epidemiology depending on time to occurrence of prosthetic joint infection: a prospective cohort study, Clin. Microbiol. Infect., 25, 353-358, https://doi.org/10.1016/j.cmi.2018.04.035, 2019.

Trojani, M.-C., Lamy, B., Ruimy, R., Amoretti, N., Risso, K., and Roux, C.: An unusual Staphylococcus saccharolyticus spondylodiscitis post kyphoplasty: a case report, BMC Infect. Diseas., 20, 539, https://doi.org/10.1186/s12879-020-05263-5, 2020.

Tunney, M. M., Patrick, S., Gorman, S. P., Nixon, J. R., Anderson, N., Davis, R. I., Hanna, D., and Ramage, G.: Improved detection of infection in hip replacements. A currently underestimated problem, J. Bone Joint Surg. Br., 80, 568-572, https://doi.org/10.1302/0301-620x.80b4.8473, 1998.

Unter Ecker, N., Koniker, A., Gehrke, T., Salber, J., Zahar, A., Hentschke, M., and Citak, M.: What Is the Diagnostic Accuracy of Alpha-Defensin and Leukocyte Esterase Test in Periprosthetic Shoulder Infection?, Clin. Orthop. Relat. Res., 477, 1712-1718, https://doi.org/10.1097/CORR.0000000000000762, 2019.

Walter, G., Vernier, M., Pinelli, P. O., Million, M., Coulange, M., Seng, P., and Stein, A.: Bone and joint infections due to anaerobic bacteria: an analysis of 61 cases and review of the literature, Eur. J. Clin. Microbiol. Infect. Dis., 33, 1355-1364, https://doi.org/10.1007/s10096-014-2073-3, 2014.

Wang, B., Toye, B., Desjardins, M., Lapner, P., and Lee, C.: A 7-year retrospective review from 2005 to 2011 of Propionibacterium acnes shoulder infections in Ottawa, Ontario, Canada, Diagn. Microbiol. Infect. Dis., 75, 195-199, https://doi.org/10.1016/j.diagmicrobio.2012.10.018, 2013.
Wang, C., Huang, Z., Li, W., Fang, X., and Zhang, W.: Can metagenomic next-generation sequencing identify the pathogens responsible for culture-negative prosthetic joint infection?, BMC Infect. Dis., 20, 253, https://doi.org/10.1186/s12879-020-049552, 2020a.

Wang, C. X., Huang, Z., Fang, X., Li, W., Yang, B., and Zhang, W.: Comparison of broad-range polymerase chain reaction and metagenomic next-generation sequencing for the diagnosis of prosthetic joint infection, Int. J. Infect. Dis., 95, 8-12, https://doi.org/10.1016/j.ijid.2020.03.055, 2020b.

Weaver, A. A., Hasan, N. A., Klaassen, M., Karathia, H., Colwell, R. R., and Shrout, J. D.: Prosthetic joint infections present diverse and unique microbial communities using combined wholegenome shotgun sequencing and culturing methods, J. Med. Microbiol., 68, 1507-1516, https://doi.org/10.1099/jmm.0.001068, 2019.

Weigelt, L., Plate, A., Stadler, L., Sutter, R., Frustaci, D., Zbinden, R., Zingg, P. O., Gerber, C., and Achermann, Y.: Alpha-defensin lateral flow test does not appear to be useful in predicting shoulder periprosthetic joint infections, Int. Orthop., 44, 1023-1029, https://doi.org/10.1007/s00264-020-04532-x, 2020.

Wildeman, P., Brüggemann, H., Scholz, C. F., Leimbach, A., and Soderquist, B.: Propionibacterium avidum as an Etiological Agent of Prosthetic Hip Joint Infection, PLoS One, 11, e0158164, https://doi.org/10.1371/journal.pone.0158164, 2016.

Xie, K., Qu, X., and Yan, M.: Procalcitonin and $\alpha$-Defensin for Diagnosis of Periprosthetic Joint Infections, J. Arthroplast., 32, 1387-1394, https://doi.org/10.1016/j.arth.2016.10.001, 2017.

Zappe, B., Graf, S., Ochsner, P. E., Zimmerli, W., and Sendi, P.: Propionibacterium spp. in prosthetic joint infections: a diagnostic challenge, Arch. Orthop. Trauma Surg., 128, 1039-1046, https://doi.org/10.1007/s00402-007-0454-0, 2008.

Zeller, V., Ghorbani, A., Strady, C., Leonard, P., Mamoudy, P., and Desplaces, N.: Propionibacterium acnes: an agent of prosthetic joint infection and colonization, J. Infect., 55, 119-124, https://doi.org/10.1016/j.jinf.2007.02.006, 2007.

Zeller, V. A., Letembet, V. A., Meyssonnier, V. A., Heym, B., Ziza, J. M., and Marmor, S. D.: Cutibacterium (Formerly Propionibacterium) avidum: A Rare but Avid Agent of Prosthetic Hip Infection, J. Arthroplast., 33, 2246-2250, https://doi.org/10.1016/j.arth.2018.02.008, 2018.

Zhang, A. L., Feeley, B. T., Schwartz, B. S., Chung, T. T., and Ma, C. B.: Management of deep postoperative shoulder infections: is there a role for open biopsy during staged treatment?, J. Shoulder Elbow Surg., 24, 15-20, https://doi.org/10.1016/j.jse.2014.04.007, 2015.

Zimmerli, W., Trampuz, A., and Ochsner, P. E.: Prostheticjoint infections, N. Engl. J. Med., 351, 1645-1654, https://doi.org/10.1056/NEJMra040181, 2004. 